\title{
Parasitosis Intestinal En Una Población De 5 A 14 Años Que Acuden A Unidades Educativas Escuelas Colegios Públicos De La Ciudad De Riobamba
}

\author{
Sandra Noemí Escobar Arrieta \\ Grupo de Investigación Leishmaniosis y otras Parasitosis en el Ecuador \\ "LEISHPAREC-ESPOCH", \\ Magister en Bioquímica Clínica, \\ Magister en Salud Pública, \\ Docente de la Escuela Superior Politécnica de Chimborazo \\ Verónica Mercedes Cando Brito \\ Magister en Farmacia Clínica y Hospitalaria \\ Docente de la Escuela Superior Politécnica de Chimborazo Farmacia \\ Carlos Eduardo Espinoza Chavez \\ Magister en Bioquímica Clínica, \\ Docente de la Escuela Superior Politécnica de Chimborazo \\ Luis Elias Guevara Iñiguez \\ Magister de Ciencias en Biotecnología, \\ Docente de la Escuela Superior Politécnica de Chimborazo
}

Doi: 10.19044/esj.2017.v13n30p11 URL:http://dx.doi.org/10.19044/esj.2017.v13n30p11

\begin{abstract}
The purpose of the current research was to determine the prevalence and risk factors linked to intestinal parasites in a population aged from 5 to 14 years who attend to public schools and high schools from Riobamba city, Riobamba-Chambo district. The prospection was carried out between October and November 2015. The population studied was identified and classified according to their age, gender, and educational institution they belong to. In addition, the relation of risk factors linked to the identified parasites was estimated. For this, it was necessary to apply a coprology examination to 2020 boys, girls and teenagers. The fecal samples collected were analyzed using the fresco technique with lugol and saline solution at $0.85 \%$. The total intestine parasite prevalence found was $35.1 \%$. The highest parasite prevalence was detected in groups aged $5(12.84 \%)$, in groups aged $8(11.71 \%)$ and in groups aged $11(11.56 \%)$, while Combatientes de Tapi Educational Unit registered a $34 \%$ in both genders. Any significant parasite difference was found among $\mathrm{p}<0.05$ ages. Nine parasite types were identified
\end{abstract}


with Entamoeba coli prevalence (26\%) Entamoeba histolytic (9.3\%), Iodamoeba bütschlii (0.8\%), Giardia lambia (4.3\%), Chilomastix mesnilii $(0.9 \%)$, Hymenolepis nana (1.4\%), Ascaris lumbricoides $(0.3 \%)$ Trichuris trichiura $(0.5 \%)$, Endolimax nana $(1.6 \%)$, from which protozoan were the predominant, some poly parasite samples were also found, it was determined that the sample studied reflects a high prevalence of intestinal parasites.

Keywords: Parasite prevalence, Risk factor, Intestinal parasites

\section{Resumen}

El objetivo de la presente investigación, fue determinar la prevalencia y factores de riesgo asociados a la parasitosis intestinal en una población de 5 a 14 años que acuden a las escuelas y colegios públicos de la ciudad de Riobamba, Distrito Riobamba-Chambo. La prospección se llevó a cabo entre los meses de octubre y noviembre del 2015. La población fue identificada y clasificada por grupos de edad, género e institución educativa, estimando la relación de los factores de riesgo asociados a los parásitos identificados. Para ello, se realizó un examen coprológico a 2020 niños, niñas y adolescentes. Las muestras fecales recolectadas fueron analizadas usando la técnica en fresco con lugol y solución salina $0.85 \%$. La prevalencia total encontrada de parasitosis intestinal fue de $35.1 \%$. La mayor prevalencia de parasitosis se dió en los grupos de edades de 5 años (12.84\%), 8 años (11.71\%) y 11 años (11.56\%), mientras que en la Unidad Educativa Combatientes de Tapi (34\%) en ambos géneros. No se observaron diferencias significativas de parasitosis entre las edades $(\mathrm{p}<0.05)$. Se identificaron nueve tipos de parásitos con prevalencias de Entamoeba coli (26\%), Entamoeba histolytica (9.3\%), Iodamoeba bütschlii (0.8\%), Giardia lamblia (4.3\%), Chilomastix mesnilii $(0.9 \%)$, Hymenolepis nana (1.4\%), Ascaris lumbricoides $(0.3 \%)$, Trichuris trichiura $(0.5 \%)$, Endolimax nana (1.6\%). De los cuales los protozoos tuvieron mayor predominio y se encontraron también muestras poliparasitadas, determiando que la muestra objeto de estudio presenta una alta prevalencia de parasitosis intestinal.

Palabras claves: Prevalencia de Parasitosis, Factores de Riesgo, Parasitosis Intestinal

\section{Introducción}

En países subdesarrollados, las malas condiciones higiénicas, la escasa cultura médica, el deficiente saneamiento ambiental y las pobres condiciones socioeconómicas están asociadas directamente con la presencia, persistencia y diseminación de parásitos intestinales, así como con las características geográficas y ecológicas específicas de cada lugar, la pobreza 
y las deficientes condiciones sanitarias derivadas de ella. Todo ello ha permitido observar una correlación directa entre la desigualdad social y el crecimiento de diversas patologías infecciosas como son la parasitosis u otras como la desnutrición, siendo uno de los estratos más afectados el conformado por infantes y jóvenes (Solano, 2008).

Uno de los parásitos que ataca frecuentemente a personas de bajos recursos económicos y tiene una amplia distribución en todo el mundo es el Ascaris lumbricoides, ya que presentan mayores casos de infecciones asintomáticos e incremento del número en el intestino, causa serias patologías como obstrucción intestinal, pancreatitis, etc. (Tomohissa, 2013).

Debido a las asombrosas capacidades que presentan los parásitos al adaptarse rápidamente a cambios del medio ambiente. Los parásitos unicelulares como los protozoos o pluricelulares como los helmintos, obtienen gran resistencia y adaptación que les permite sobrevivir en condiciones ambientales que los destruirían de no adquirir estas capacidades como la de los vectores u hospedadores.

Durante la fase de colonización, la supervivencia del parásito patógeno depende no solo de la habilidad de colonizar, sino también de la habilidad de neutralizar los mecanismos de defensa del organismo al cual coloniza. Así, la patogenicidad o virulencia de los parásitos refleja la interacción dinámica entre ellos y el hospedador y su capacidad de respuesta a los sistemas defensivos, condición necesaria para la supervivencia parasitaria y el mantenimiento y/o transmisión de la infección (Lujan, 2006)

Según Casquina y Martínez (2011) en el Perú, la parasitosis intestinal es una de las 10 principales causas de muerte, constituyendo un problema de salud con un $7.7 \%$, lo que significa que uno de cada tres personas porta al menos una especie de parasito en el intestino, de los cuales la niñez es el grupo más vulnerable debido a su nivel inmunológico bajo y amplio contacto con los agentes infecciosos.

Según los estudios y datos recolectados por regiones de este país indican que en la región de la selva o amazónica existe un predominio de helmintiasis y en regiones como sierra y costa existe un predominio de protozoarios.

En Ecuador, al igual que en otros países de Latinoamérica, los aspectos relacionados con las infecciones parasitarias continúan siendo un importante problema de salud pública (Halpenny, 2013). En muchas ocasiones es difícil determinar si las infecciones son consecuencia directa de la desnutrición, por los efectos que éstas provocan sobre la inmunidad de los individuos, o si por el contrario dicha desnutrición es una consecuencia más de la propia infección parasitaria. Estudios han puesto en manifiesto la importancia de estas infecciones con porcentajes de helmintiasis y 
protozoosis que en ocasiones han llegado a afectar al 90\% de la población infantil (Sackey, 2014).

En el estudio que realizó Jacobsen (2007), observó que la mejora de las instalaciones sanitarias no aseguraba por sí solas, que las comunidades eviten el riesgo de la transmisión de estas infecciones parasitarias, por lo que son necesarias otras medidas formativas complementarias. Entre los principales daños que pueden producir las infecciones por helmintos se encuentran los de tipo nutricional-diarreicas, pérdidas de apetito, síndromes de mala absorción, afectando principalmente al estado cognitivo y de desarrollo del niño/a, entre otras (Cepon-Robins, 2014). Es por este motivo, que son necesarias medidas directamente enfocadas a la población infantil, ya que estas infecciones son de difícil tratamiento e incluso ocasionan daños irreversibles a largo plazo.

Otros factores importantes que se han considerado dentro de la transmisión de parasitosis intestinal en las escuelas y colegios públicos de Riobamba, son la falta de saneamiento que existe en las cisternas de agua de consumo humano de las instituciones, siendo este, un factor importante en la transmisión de parásitos y otras enfermedades. También influye la falta de control sanitario a los puestos de comidas ambulantes que venden cerca de las instituciones, donde muchos de los estudiantes consumen alimentos que ahí se expenden, sin saber el tratamiento y la procedencia que tuvieron estos alimentos antes de llegar a ser consumidos por ellos.

Por lo antes expuesto el presente proyecto de investigación se enfocó en determinar la prevalencia y factores de riesgo asociados a los parásitos más frecuentes en la población de niños y adolescentes con edades comprendidas entre los 5 años y 14 años, los mismos que acuden a las Unidades Educativas, Escuelas y Colegios Públicos de la ciudad de Riobamba (Distrito Riobamba- Chambo). Para tal finalidad se trabajó conjuntamente con el proyecto de investigación perteneciente a la línea clínica de diagnóstico de enfermedades parasitarias a cargo del Grupo de Investigación LEISHPAREC (Leishmaniosis y otras parasitosis en Ecuador).

\section{Metodología}

La Dirección del Ministerio de Educación Distrito Riobamba Chambo contribuyó con el apoyo administrativo (permisos de ingreso a las instituciones) para la realización del proyecto. La Universidad de Granada aportó con el asesoramiento de varios de sus profesores del Departamento de Parasitología, Facultad de Farmacia (España).

La investigación fue realizada en la carrera de Bioquímica y Farmacia, Facultad de Ciencias de la Escuela Superior Politécnica de Chimborazo (ESPOCH), donde se facilitaron las instalaciones para el examen parasitológico y derivados de la investigación. Así también 
contribuyó el equipo profesional representado por los liderada por estuvo a cargo de los miembros del Grupo de Investigación LEISHPAREC (Leishmaniosis y otras parasitosis en Ecuador).

La población de la investigación está representada por las principales unidades educativas de Riobamba, entre ellas: Combatientes de Tapi, Camilo Gallegos Toledo, Leonidas Gracia Ortiz y Pedro Vicente Maldonado, mientras que la muestra representa a 2020 niños y adolescentes de 5 a 14 años que acuden a las Unidades Educativas, Escuelas y Colegios Públicos de la ciudad de Riobamba, 2015.

Previo al estudio de investigación de parasitosis hubo la necesidad de tramitar los permisos legales en el Ministerio de Educación, organismo responsable que otorga los permisos de ingreso a las unidades educativas, Ya en las instituciones educativas se socializó las razones, el objetivo y los procedimiento de recolección de muestras en presencia de rectores, director, inspectores y estudiantes. Culminada la explicación y despejadas las inquietudes, se procedió a entregar los materiales de recolección de muestras de heces y una encuesta a cada estudiante que debió ser entregadas el siguiente día a los miembros de la investigación.

La recolección de las muestras de heces fueron alojados en un cooler con su respectivo registro y codificación numérica y procesadas en el laboratorio de parasitología de la Facultad de Ciencias de la ESPOCH. Para el análisis de las muestras, se prepararon los materiales y soluciones necesarios como: solución salina (Suero fisiológico), lugol, placas porta objetos, placas cubre objetos, palillos y microscopio óptico marca Olympus. Una vez codificado cada placa porta objetos, se procedió a colocar una gota de solución salina y una gota de lugol en la placa porta objetos. Mediante la ayuda de un palillo se procedió a homogeneizar y coger una pequeña cantidad de muestra, la misma que se la colocó en las dos soluciones. Posteriormente se colocó la placa preparada con la muestra sobre la platina del microscopio, se enfocó y se procedió hacer una revisión de cada campo en cada una de las soluciones (solución salina y lugol). Los resultados de los parásitos identificados fueron registrados y transcritos a una base de datos conjuntamente con su respectiva encuesta, que posteriormente fueron analizados en el programa estadístico SPSS statistics. Y finalmente se analizó: la prevalencia total de parasitosis, la prevalencia de cada parásito, la prevalencia asociado por género, localización geográfica y sus respectivos factores de riego, mediante el uso del programa estadístico SPSS STATISTICS. 


\section{Resultados}

A continuación se presentan los resultados obtenidos

Cuadro 1. Prevalencia de parasitismo en la muestra de estudio.

\begin{tabular}{|c|c|c|}
\hline & Número de casos & Porcentaje (\%) \\
\hline Positivo & 709 & 35,1 \\
\hline Negativo & 1263 & 62,5 \\
\hline Datos perdidos & 48 & 2,4 \\
\hline Total & 2020 & 100 \\
\hline
\end{tabular}

Fuente: Analisis de laboratorio de muestras de heces

Realizado por: Autores, 2017

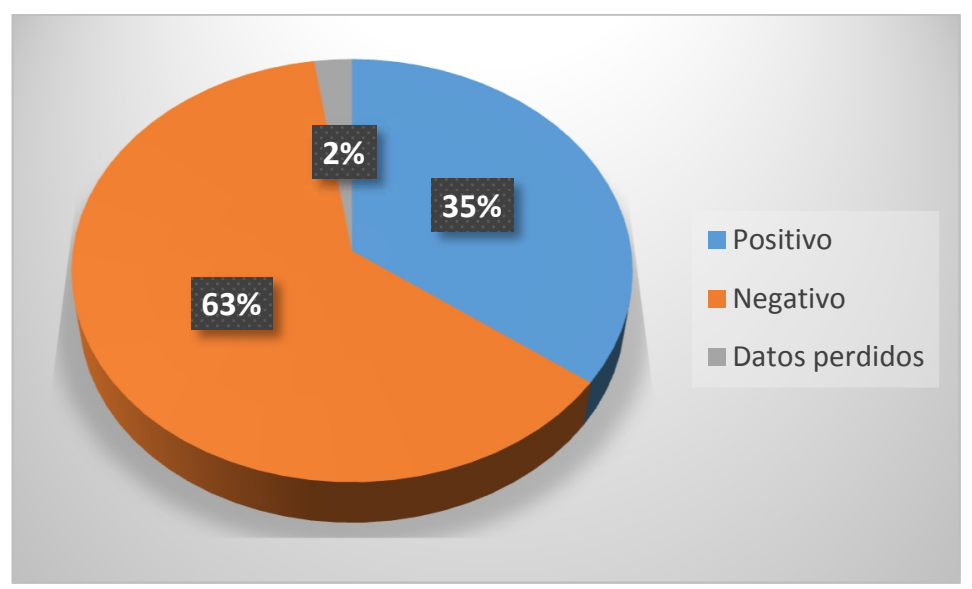

Gráfica 1. Prevalencia de parasitismo en la muestra de estudio.

\section{Análisis}

En relación a los resultados representados en el cuadro 1, el 35.1\% de la población en estudio registró positivo a la presencia de parasitosis intestinal, lo que significa que aproximadamente un tercio de la población estudiada, fue infectada al menos por un tipo de parasito, estos datos fueron similares a los resultados obtenidos por Rinne, S. en un estudio de parasitosis realizado en una comunidad de las tierras altas del Ecuador (Rinne, 2005). Nuestros resultados generales evidencian que las parasitosis intestinales en las Unidades Educativas del Cantón Riobamba suponen un importante problema de Salud Pública.

Cuadro 2. Monoparasitosis (infección por una única especie) y Poliparasitosis (dos o más especies de parásitos).

\begin{tabular}{cc}
\hline & Número de casos \\
\hline MONOPARASITOSIS $=1$ & 532 \\
POLIPARASITOSIS $\geq 2$ & 177 \\
Total & 709 \\
\hline
\end{tabular}

Fuente: Analisis de laboratorio de muestras de heces

Realizado por: Autores, 2017 


\section{Análisis}

En relación a los resultados representados en el cuadro 2, se registró que el $75 \%$ de la población infectada estaba colonizada por un solo tipo de parasito, pero también existe una cantidad significativa de poliparasitosis, por lo que es un factor a tomar en consideración, ya que es un indicativo de deficientes hábitos de higiene, saneamiento de agua y control sanitario en los alimentos que son expendidos y consumidos en el interior y exterior de las instituciones educativas por los alumnos, los cuales están expuestos constantemente a infecciones parasitarias. Al comparar estos resultados con los obtenidos por Valverde, C. y Moreno M, se encontró que existe mayor porcentaje de monoparasitosis en nuestro estudio y menor de poliparasitosis.

Cuadro 2. Parásitos más prevalentes en la muestra de estudio.

\begin{tabular}{ccc}
\hline TIPO DE PARASITO & \multicolumn{2}{c}{ Positivos } \\
& $\mathrm{N}^{\circ}$ & $\%$ \\
\hline Quistes de Entamoeba coli & 525 & 26.6 \\
Quistes de Entamoeba histolytica & 187 & 9.5 \\
Trofozoito de Entamoeba histolytica & 1 & 0.1 \\
Quistes de Iodamoeba bütschlii & 16 & 0.8 \\
Quistes de Endolimax nana & 33 & 1.7 \\
Huevo de Hymenolepis nana & 28 & 1.4 \\
Quistes de Giardia lamblia & 86 & 4.4 \\
Quistes de Chilomastix mesnilii & 18 & 0.9 \\
Huevo de Ascaris lumbricoides & 6 & 0.3 \\
Huevo de Trichuris trichiura & 1 & 0.1 \\
\hline
\end{tabular}

Fuente: Analisis de laboratorio de muestras de heces

Realizado por: Autores, 2017

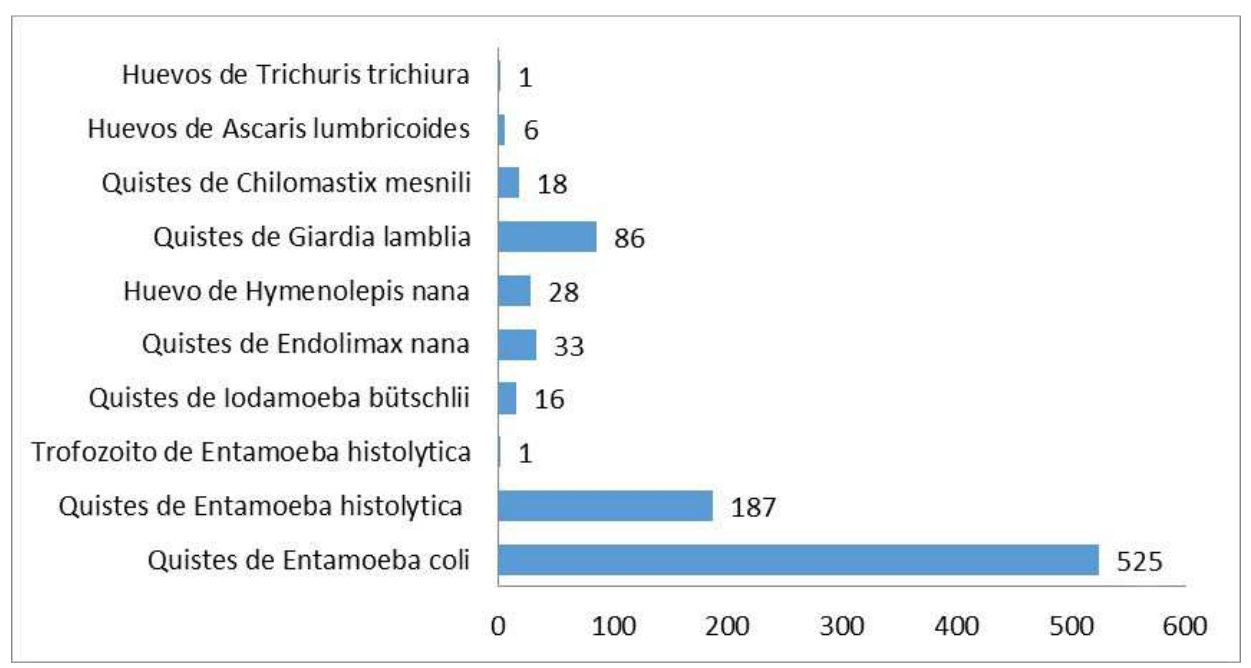

Gráfica 2. Parásitos más prevalentes en la muestra de estudio. 


\section{Análisis}

Con base a los resultados representados en el Cuadro 2, se observó que los parásitos diagnosticados son de mayor a menor prevalencia: Quistes de Entamoeba coli (26.6\%), Quistes de Entamoeba histolytica (9.5\%), Quistes de Giardia lamblia (4.4\%), Quistes de Endolimax nana (1.7\%), Huevo de Hymenolepis nana (1.4\%), Quistes de Chilomastix mesnilii $(0.9 \%)$, Quistes de Iodamoeba bütschlii (0.8\%), Huevos de Ascaris lumbricoides $(0.3 \%)$, Trofozoito de Entamoeba histolytica (0.1\%), Huevos de Trichuris trichiura $(0.1 \%)$. Estos resultados fueron comparados con los obtenidos en la investigación de Jacobsen, H, y mostró que existe un menor porcentaje de parasitosis en la presente investigación (Jacobsen, 2007).

Cuadro 3. Edades con mayor prevalencia de parasitosis en la muestra de estudio.

\begin{tabular}{ccc}
\hline \multirow{2}{*}{ EDAD } & \multicolumn{2}{c}{ CASOS PARASITOSIS } \\
& Positivo & Negativo \\
\hline 3 & 8 & 22 \\
4 & 32 & 65 \\
5 & 91 & 158 \\
6 & 59 & 123 \\
7 & 70 & 129 \\
8 & 83 & 127 \\
9 & 79 & 115 \\
10 & 68 & 106 \\
11 & 82 & 122 \\
12 & 58 & 131 \\
13 & 43 & 82 \\
14 & 27 & 74 \\
15 & 9 & 9 \\
\hline
\end{tabular}

Fuente: Analisis de laboratorio de muestras de heces

Realizado por: Autores, 2017

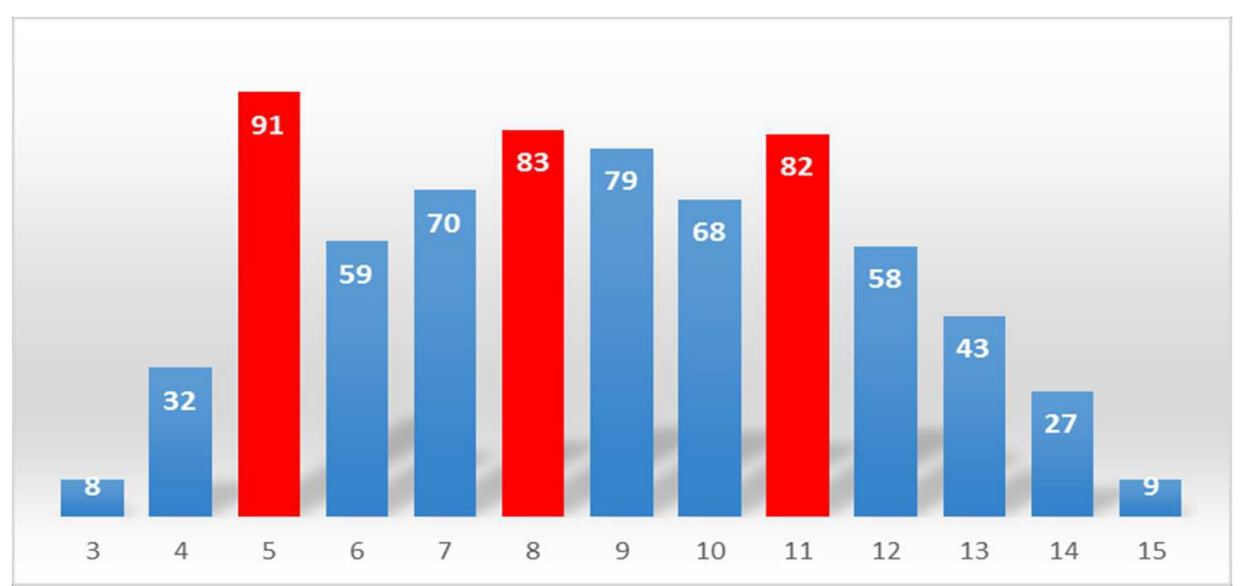

Gráfica 3. Edades con mayor prevalencia de parasitosis en la muestra de estudio. 


\section{Análisis}

Con base a los resultados registrados en el cuadro 3, se encontró que los parásitos más frecuentes en la población parasitada se distribuyen con mayor proporción en las edades de 5, 8 y 11 años, lo cual puede estar relacionado a que en estas edades existe mayor contacto con los agentes infecciosos y por la inmadurez del sistema inmune como se ha indicado en un estudio realizado por Casquina, L \& Martínez, E., Perú, 2011. El cual cita dentro de su investigacion a (Botero \& Restrepo, 2003), donde indica que el sistema inmune durante estos años, no responde efectivamente como un sistema inmune maduro frente a estos organismos infecciosos. (Casquina, 2011).

Cuadro 4. Género (niño-niña) con mayor prevalencia (número de casos) de parásitos en la muestra de estudio.

\begin{tabular}{cccc}
\hline & & \multicolumn{2}{c}{ CASOS PARASITOSIS } \\
& & Positivo & Negativo \\
\hline GENERO & Niño & 393 & 705 \\
& Niña & 316 & 558 \\
\hline
\end{tabular}

Fuente: Analisis de laboratorio de muestras de heces

Realizado por: Autores, 2017

\section{Análisis}

Acorde al análisis estadístico de los resultados obtenidos que se muestran en el Cuadro 4, no existen diferencias estadísticamente significativas de parasitosis entre niños y niñas, lo que significa que ambos géneros son igualmente susceptibles de infectarse por algún tipo de parasito, estando igualmente expuestos a los factores predisponentes. Con los resultados obtenidos en la investigación de (Sánchez \& Rojas , 2011), donde tampoco existe diferencia alguna de parasitosis por géneros.

Cuadro 5. Prevalencia de parasitosis en la muestra de estudio.

\begin{tabular}{ccc}
\hline DIRECCIÓN & \multicolumn{2}{c}{ CASOS PARASITOSIS } \\
& Positivo & Negativo \\
\hline UE. COMBATIENTES DE TAPI & 230 & 501 \\
UE. CAMILO GALLEGOS TOLEDO & 90 & 127 \\
UE. LEONIDAS GRACIA ORTIZ & 186 & 299 \\
UE. PEDRO VICENTE MALDONADO & 181 & 310 \\
\hline
\end{tabular}

Fuente: Analisis de laboratorio de muestras de heces

Realizado por: Autores, 2017 


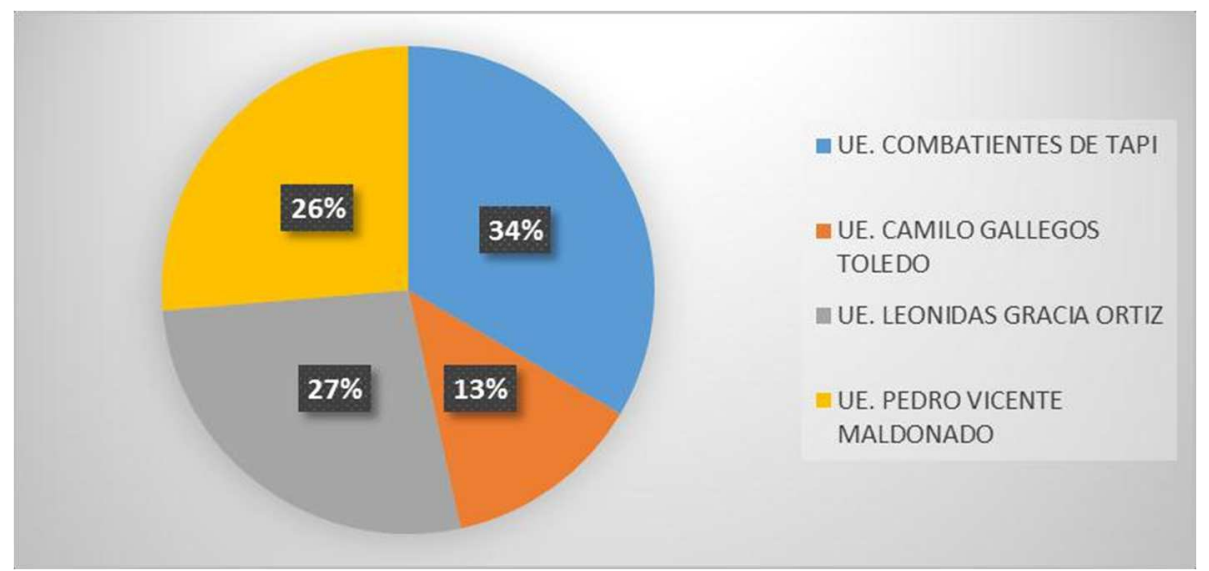

Gráfica 4. Prevalencia de parasitosis en la muestra de estudio.

\section{Análisis}

Con base a los resultados representados en el cuadro 5, se encontró que los parásitos con mayor prevalencia en la población estudiada, se encuentran en mayor proporción en la Unidad Educativa Combatientes de Tapi (34\%), Unidad Educativa Leónidas García Ortiz (27\%), Unidad Educativa Pedro Vicente Maldonado (26\%) y la Unidad Educativa Camilo Gallegos Toledo (13\%). Ello nos permite atribuir a factores como la falta de control sanitario en los puestos de comida, al interior y exterior de las instituciones, inadecuada higiene personal, falta de control de saneamiento del agua de consumo humano de las instituciones y condiciones higiénicas de los lugares donde juegan estos niños (lugares de tierra). Todos los factores mencionados pueden estar implicados en la infección por parásitos de estos niños.

Cuadro 6. Tipo de parasitosis según las instituciones educativas.

\begin{tabular}{cccccccccc}
\hline & QA & QA & QCh & Q & QG & HH & HA & QE & HT \\
& C & H & M & I & L & N & L & N & T \\
\hline UE. COMBATIENTES DE TAPI & 162 & 61 & 4 & 8 & 26 & 5 & 1 & 12 & 0 \\
UE. CAMILO GALLEGOS & 68 & 23 & 2 & 1 & 10 & 8 & 1 & 4 & 0 \\
TOLEDO & 154 & 42 & 5 & 3 & 19 & 8 & 4 & 0 & 1 \\
$\begin{array}{c}\text { UE. LEONIDAS GRACIA ORTIZ } \\
\text { UE. PEDRO VICENTE }\end{array}$ & 128 & 53 & 7 & 4 & 27 & 3 & 0 & 16 & 0 \\
MALDONADO & & & & & & & & & \\
\hline
\end{tabular}

Fuente: Encuestas aplicadas a los estudiantes

Realizado por: Autores, 2017

Equivalencia de especies: QAC: Quistes de Entamoeba coli; QAH: Quistes de Entamoeba histolytica; QChM: Quistes de Chilomastix mesnilii; QI: Quistes de Iodamoeba bütschlii; QGL: Quistes de Giardia lamblia; HHN: Huevo de Hymenolepis nana; HAL: Huevo de Ascaris lumbricoides; QEN: Quistes de Endolimax nana; HTT: Huevo de Trichuris trichiura. 


\section{Análisis}

En el análisis de los resultados registrados en el cuadro 6, se evidenció que en la Unidad Educativa Combatientes de Tápi las infecciones más frecuentes en los niños se dieron por Entamoeba coli, Entamoeba histolytica, Iodamoeba bütschlii, Giardia lamblia; mientras que en las Unidades Educativas Leónidas García Ortiz, Pedro Vicente Maldonado y Camilo Gallegos Toledo fueron diagnosticados en menor proporción Chilomastix mesnilii, Endolimax nana, Hymenolepis nana y Ascaris lumbricoides.

\section{Riesgo Estimado en función de los valores porcentuales obtenidos}

Cuadro 7. Cuadro de relación al ambiente donde viven los niños y su relación con parasitosis.

\begin{tabular}{ccccc}
\hline \multirow{2}{*}{ AMBIENTE VIVIENDA } & \multicolumn{3}{c}{ CASOS DE PARASITOSIS } \\
& Positivo & \multicolumn{2}{c}{ Negativo } \\
\hline Periurbano & 60 & $3.0 \%$ & 72 & $3.7 \%$ \\
Rural & 208 & $10.5 \%$ & 316 & $16.0 \%$ \\
Urbano & 441 & $22.4 \%$ & 875 & $44.4 \%$ \\
\hline
\end{tabular}

Fuente: Encuestas aplicadas a los estudiantes

Realizado por: Autores, 2017

\section{Análisis}

Con base a los resultados registrados en el cuadro 7, de los 709 casos positivos, se encontró que más de la mitad de esta población vive en el sector urbano seguidos por los que viven en el sector rural. Esto indica que los niños que viven en la ciudad presentan el doble de parasitación en comparación con los niños del sector urbano, corroborando la existencia de variación en la infección parasitaria en función del tipo de ambiente donde viven los infantes, como indicaron (Casquina, 2011). En su estudio de parasitosis donde cita a (Ayaqui \& Huanca, 2000). El medio rural se ha identificado como un entorno más propicio para la transferencia de estas infecciones, por lo que serían necesarios nuevos estudios en profundidad que permitiesen identificar a aquellos elementos que hacen que los niños urbanos se infecten con mayor intensidad.

Cuadro 8. Cuadro de relación al lugar donde defecan y su relación con parasitosis.

\begin{tabular}{|c|c|c|c|c|}
\hline \multirow[t]{2}{*}{ LUGAR DONDE DEFECA } & \multicolumn{4}{|c|}{ CASOS DE PARASITOSIS } \\
\hline & \multicolumn{2}{|c|}{ Positivo } & \multicolumn{2}{|c|}{ Negativo } \\
\hline Letrina & 13 & $0.7 \%$ & 21 & $1.1 \%$ \\
\hline Pozo séptico & 34 & $1.7 \%$ & 46 & $2.3 \%$ \\
\hline Baño & 662 & $33.6 \%$ & 1196 & $60.6 \%$ \\
\hline
\end{tabular}

Fuente: Encuestas aplicadas a los estudiantes

Realizado por: Autores, 2017 


\section{Análisis}

Según los resultados registrados en el cuadro 8, de los 709 niños que dieron positivo a presencia de parásitos, el 33.6\% defecan en baño ubicado en sus casas, lo que podría ser un indicativo de malos hábitos de higiene personal en sus domicilios, así como limpieza de los baños. El análisis estadístico tampoco ha mostrado la existencia de diferencias estadísticamente significativas en función del lugar donde defecan los niños.

Cuadro 9. Cuadro de relación al lavado de manos después de defecar y su relación con parasitosis.

\begin{tabular}{ccccc}
\hline LIMPIEZA DE MANOS DESPUES DE & \multicolumn{3}{c}{ CASOS DE PARASITOSIS } \\
DEFECAR & \multicolumn{2}{c}{ Positivo } & \multicolumn{2}{c}{ Negativo } \\
\hline No & 12 & $0.6 \%$ & 21 & $1.1 \%$ \\
Si sin jabón & 84 & $4.3 \%$ & 142 & $7.2 \%$ \\
Si con jabón & 613 & $31.1 \%$ & 1100 & $55.8 \%$ \\
\hline
\end{tabular}

Fuente: Encuestas aplicadas a los estudiantes

Realizado por: Autores, 2017

\section{Análisis}

Con base a los resultados resgitrados en el Cuadro 9, el 31.1\% de la población total que dieron positivo a la presencia de parasitosis, se lava las manos después de defecar con jabón, pero a la vez presentan una alta parasitosis. Estos resultados podrían indicar la existencia de un sesgo de clasificación en la población relacionado con que al completar sus encuestas, las personas se viesen influenciadas por motivos relacionados con la imagen familiar, ya que, lo esperable debería ser que en el grupo (lavado de manos con jabón después de defecar) las parasitosis fuesen más bajas. Otro elemento que podría haber afectado a estos resultados, es la importante diferencia de niños que se lavan las manos respecto a los que no lo hacen. Sin embargo, el elevado porcentaje de niños negativos $(63.0 \%)$ identificados en el grupo que se lavan las manos después de defecar, avalan la teoría de que este hábito es un importante factor de protección, porque es un indicativo de una reinfección oral-fecal.

Cuadro 10. Cuadro de relación al lavado de manos antes de comer y su relación con parasitosis.

\begin{tabular}{ccccc}
\hline \multirow{2}{*}{ LIMPIEZA DE MANOS ANTES DE COMER } & \multicolumn{3}{c}{ CASOS DE PARASITOSIS } \\
& \multicolumn{2}{c}{ Positivo } & \multicolumn{2}{c}{ Negativo } \\
\hline No & 20 & $1.0 \%$ & 36 & $1.8 \%$ \\
Si sin jabón & 117 & $5.9 \%$ & 176 & $8.9 \%$ \\
Si con jabón & 572 & $29.0 \%$ & 1051 & $53.3 \%$ \\
\hline
\end{tabular}

Fuente: Encuestas aplicadas a los estudiantes

Realizado por: Autores, 2017 


\section{Análisis}

De los resultados que se registran en el Cuadro 10, se encontró que el $34.9 \%$ de la población que dio positivo a la presencia de parasitosis, se lavan las manos antes de comer; pero a la vez presentan una alta parasitosis. Nuevamente se detectó un posible sesgo de clasificación como fue indicado en el anterior apartado. Esto podría indicar que los niños son propensos a reinfectarse, a la predominancia y falta de higiene personal. Del mismo modo, se continuó observando un importante porcentaje de niños (62.2\%) no parasitados que se lavan las manos antes de las comidas.

Cuadro 11. Cuadro de relación de como están las uñas de esta población y su relación con parasitosis.

\begin{tabular}{ccccc}
\hline COMO ESTAN SUS UÑAS & \multicolumn{3}{c}{ CASOS DE PARASITOSIS } \\
& \multicolumn{2}{c}{ Positivo } & \multicolumn{2}{c}{ Negativo } \\
\hline Largas sucias & 6 & $0.3 \%$ & 11 & $0.6 \%$ \\
Largas limpias & 61 & $3.1 \%$ & 90 & $4.6 \%$ \\
Cortas & 642 & $32.6 \%$ & 1162 & $58.9 \%$ \\
\hline
\end{tabular}

Fuente: Encuestas aplicadas a los estudiantes

Realizado por: Autores, 2017

\section{Análisis}

En los resultados obtenidos que se representan en el Cuadro 11, se encontró que el $32.6 \%$ de la población que registraron positivo a la presencia de parasitosis tienen uñas cortas. Las mismas consideraciones efectuadas en los Cuadros 9 y 10 sería aplicable a este caso en lo relativo a sesgo de clasificación y tamaño de la muestra. Nuevamente las uñas cortas parece actuar como elemento de protección con un 58.9\% de los niños no parasitados en dicho grupo.

Cuadro 12. Cuadro de relación al juegar en zonas de tierra.

\begin{tabular}{ccc}
\hline & JUEGA EN ZONAS DE TIERRA & $\mathrm{Si}$ \\
Especie & No & 91 \\
QAH & 96 & 10 \\
QI & 6 & 47 \\
QGL & 39 & 16 \\
HHN & 12 & 5 \\
HAL & 1 & 0 \\
HTT & 1 & 1 \\
TrzAH & 0 & 11 \\
QEN & 22 & 6 \\
QChM & 12 & \\
\hline
\end{tabular}

Fuente: Encuestas aplicadas a los estudiantes

Realizado por: Autores, 2017

Equivalencia de especies: QAH: Quistes de Entamoeba histolytica; QI: Quistes de Iodamoeba bütschlii; QGL: Quistes de Giardia lamblia; HHN: Huevo de Hymenolepis nana; HAL: Huevo de Ascaris lumbricoides; HTT: 
Huevo de Trichuris trichiura; TRZAH: Trofozoito de Entamoeba histolytica; QEN: Quistes de Endolimax nana; QChM: Quistes de Chilomastix mesnilii.

\section{Análisis}

De los resultados representados en el Cuadro 12, de toda la población que dió positivo a la presencia de parasitosis y que juegan en zonas de tierra, mayoritariamente se infectan con Quistes de Giardia lamblia, Huevos de Hymenolepis nana, así como Quistes de Iodamoeba y Huevos de Ascaris lumbricoides. Esto se debe a que las formas infectantes de estos parásitos son muy resistentes a las condiciones ambientales, lo cual corrobora los resultados Costamagna, R \& Visciarelli, E con la información que indica en su libro de parasitología (Costamagna, 2004, pp. 81-82, 107-109, 192-194). A excepción de los Quistes de Entamoeba histolytica, Huevos de Trichuris trichiura, Quistes de Endolimax nana y Quistes de Chilomastix mesnilii; todas las restantes especies diagnosticadas se han encontrado en mayor número en los niños que jugaban e en el campo.

Cuadro 13. Cuadro en relación a los síntomas más frecuentes y su relación con la parasitosis.

\begin{tabular}{ccccc}
\hline \multirow{2}{*}{ SINTOMAS } & \multicolumn{3}{c}{ CASOS PARASITOSIS } \\
& 64 & $3.2 \%$ & 99 & $5.0 \%$ \\
Fiebre & 34 & $1.7 \%$ & 45 & $2.3 \%$ \\
Vómitos & 45 & $2.3 \%$ & 51 & $2.6 \%$ \\
Diarrea & 102 & $5.2 \%$ & 175 & $8.9 \%$ \\
Cansancio & 23 & $1.2 \%$ & 37 & $1.9 \%$ \\
Fatiga & 441 & $22.4 \%$ & 856 & $43.4 \%$ \\
Asintomáticos &
\end{tabular}

Fuente: Encuestas aplicadas a los estudiantes

Realizado por: Autores, 2017

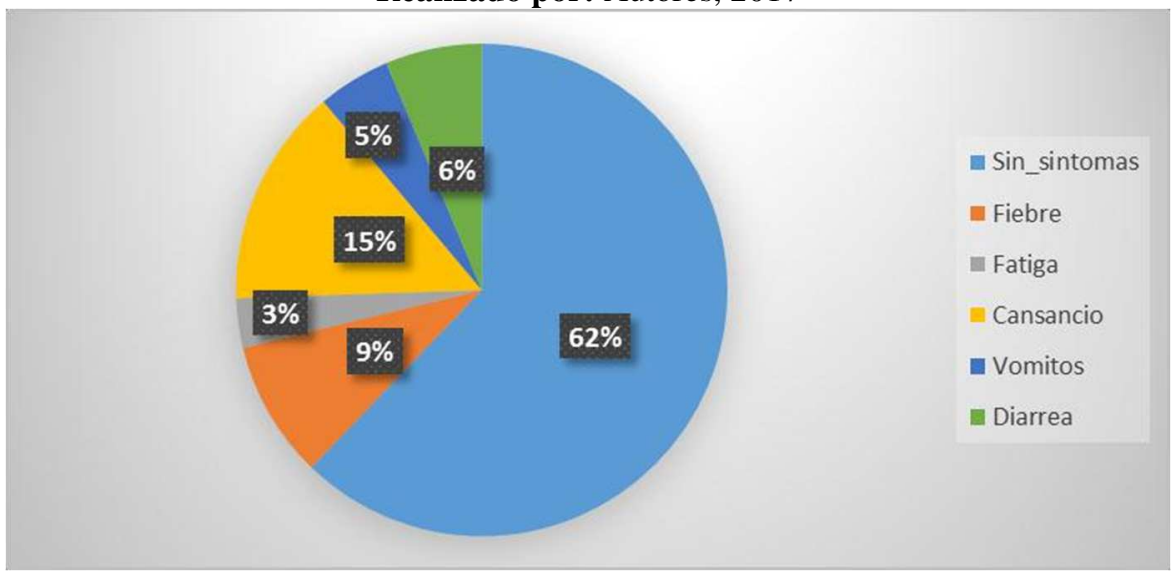

Gráfica 5. Cuadro en relación a los síntomas más frecuentes y su relación con la parasitosis.. 


\section{Análisis}

En relación a los resultados presentados en el Cuadro 13 y Gráfica 5, se encontró que el $37.8 \%$ de toda la población que diópositivo a parasitosis; presentó alguna sintomatología clínica; de los cuales fiebre, vómito y diarrea han sido ampliamente relacionados con la presencia de parásitos como lo indica (Sánchez \& Rojas, 2011) en su estudio. Esta sintomatología suele correlacionarse con infecciones persistentes por algún parasito patógeno, de entre los que han sido identificados en este estudio: Entamoeba histolytica, Giardia lamblia, Hymenolepis nana o Ascaris lumbricoides. Es importante indicar que el 22.4\% (62.2\% de la población infectada) de la población total resultó infectada y asintomática. Este resultado apoya a lo que manifiesta Vasquez, O \& Campos, T., que aún cuando la mayor parte de la población presenta algún parasito suele ser asintomática, y que sólo algunos casos presentan sintomatología durante el proceso infeccioso (Vasquez \& Campos, 2009).

Cuadro 14. Cuadro en relación al tratamiento de agua de consumo y su relación con la parasitosis.

\begin{tabular}{cccccccccccc}
\hline AGUA DE & QA & TzrA & QA & QG & HH & Q & QCh & HA & QE & HT & TOTA \\
CONSUMO & H & H & C & L & N & I & M & L & N & T & L \\
\hline Embotellada & 50 & 0 & 145 & 21 & 6 & 4 & 4 & 0 & 9 & 0 & 239 \\
Agua llave & 69 & 0 & 197 & 35 & 12 & 7 & 4 & 4 & 10 & 0 & 338 \\
Agua filtro & 14 & 1 & 36 & 11 & 2 & 1 & 0 & 1 & 3 & 0 & 69 \\
Agua cisterna & 9 & 0 & 31 & 6 & 3 & 0 & 2 & 0 & 2 & 0 & 53 \\
Agua hervida & 45 & 0 & 116 & 13 & 5 & 4 & 8 & 1 & 9 & 1 & 202 \\
\hline
\end{tabular}

Fuente: Encuestas aplicadas a los estudiantes

Realizado por: Autores, 2017

Equivalencia de especies: QAH: Quistes de Entamoeba histolytica; TRZAH: Trofozoito de Entamoeba histolytica; QAC: Quistes de Entamoeba coli; QGL: Quistes de Giardia lamblia; HHN: Huevo de Hymenolepis nana; QI: Quistes de Iodamoeba bütschlii; QChM: Quistes de Chilomastix mesnilii; HAL: Huevo de Ascaris lumbricoides; QEN: Quistes de Endolimax nana; HTT: Huevo de Trichuris trichiura.

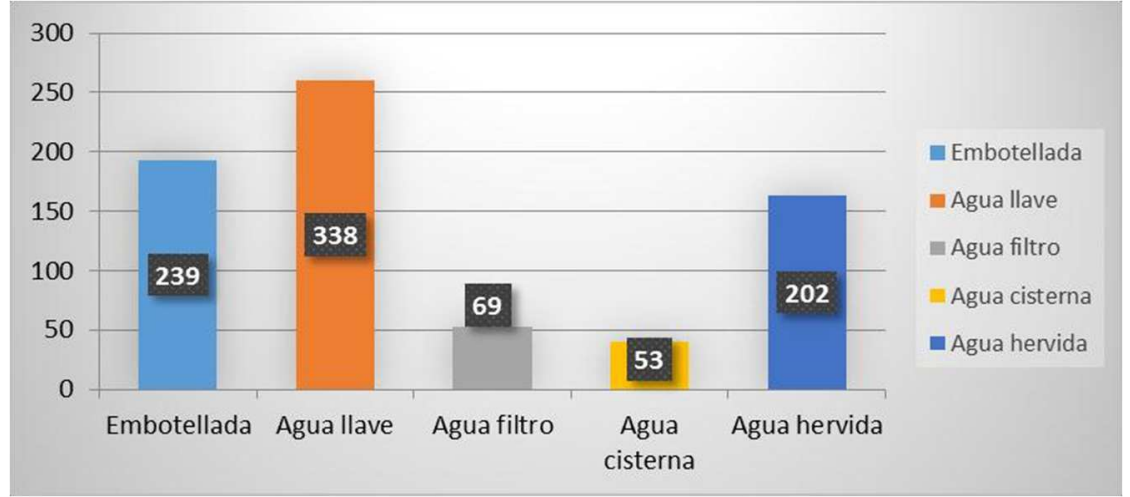

Gráfica 6. Muestra de estudio según el tratamiento de agua de consumo. 


\section{Análisis}

En relación a los resultados presentados en el Cuadro 14 y Gráfica 6, se evidencia que existe mayor presencia de parasitosis en aquellos niños que beben agua directamente de la llave, pudiendo resultar un importante factor en la transmisión de parásitos, y al mismo tiempo que el agua consumida a diario podría no ser de calidad y apta para el consumo humano. También se evidenció que hay parasitosis en cantidades menores pero significativas en niños que dicen consumir agua embotellada, lo que indica que este recurso debería ser estudiado en mayor profundidad para verificar su calidad. En las normas NTE INEN 1108 y 2200, en sus requisitos exige que para agua embotellada debe existir una ausencia total de parásitos y en los requisitos que exige para agua de consumo humano debe existir ausencia de Quistes de Giardia lamblia, por otra parte, cuando el agua es sometida a un proceso físico como hervirla los parásitos presentes en ellas deberían morir por lo que aquellos niños no deberían presentar un 23\% de parasitosis, en la Gráfica 6; se puede apreciar con más especificidad que parásitos de los identificados en esta investigación se encuentran en mayor proporción en los diferentes medios de suministro de agua (NTE INEN 1108, 2016) (NTE INEN 2200, 2016)

Cuadro 15. Cuadro en relación al tratamiento que aplica para comer fruta o verdura y su relación con la parasitosis.

\begin{tabular}{ccccc}
\hline \multirow{2}{*}{ TRATAMIENTO DE VERDURA/FRUTA } & \multicolumn{2}{c}{ CASOS DE PARASITOSIS } \\
& \multicolumn{2}{c}{ Positivo } & \multicolumn{2}{c}{ Negativo } \\
\hline No come fruta & 50 & $2.5 \%$ & 64 & $3.2 \%$ \\
Hervida & 98 & $5.0 \%$ & 142 & $7.2 \%$ \\
Lavada & 517 & $26.2 \%$ & 981 & $49.7 \%$ \\
Sin tratar & 44 & $2.2 \%$ & 76 & $3.9 \%$ \\
\hline
\end{tabular}

Fuente: Encuestas aplicadas a los estudiantes

Realizado por: Autores, 2017

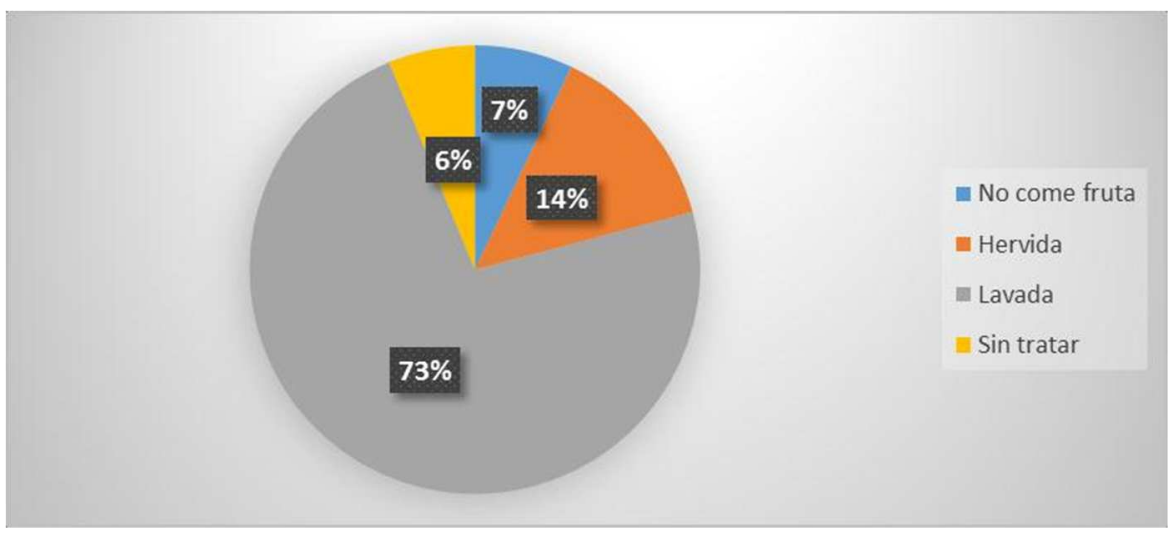

Gráfica 7. Tratamiento que aplica para comer fruta o verdura y su relación con la parasitosis. 


\section{Análisis}

En relación a los resultados representados en el Cuadro 15 y Gráfica 7, se evidencia que existe mayor presencia de parasitosis en niños que consumen fruta o verdura lavada $(26.2 \%$ del total de casos o $73.0 \%$ de los niños parasitados), siendo este elemento de transmisión muy importante como indicativo de asociación a las parasitosis. Por todo ello no es suficiente con lavar la fruta, ya que también pueden existir parásitos presentes en el agua con la que la estamos tratando y sería aconsejable que adicionalmente la fruta fuese sumergida en agua con lejía (solución de hipoclorito al 5\%) como recomiendan los expertos en salud. También se evidenció que existe parasitosis en cantidades menores pero elevadas en niños que consumen fruta o verdura de forma hervida, lo que nos indica que probablemente las temperaturas alcanzadas en los procesos de esterilización del agua por hervido no son suficientes. Estos resultados apoyan a los obtenidos en el Cuadro 15.

Cuadro 16. Relación de relación a si tienen animales en casa y su relación con la parasitosis.

\begin{tabular}{ccccc}
\hline ANIMALES EN CASA & \multicolumn{3}{c}{ CASOS PARASITOSIS } \\
& \multicolumn{2}{c}{ Positivo } & \multicolumn{2}{c}{ Negativo } \\
\hline $\mathrm{No}$ & 218 & $11.1 \%$ & 418 & $21.2 \%$ \\
$\mathrm{Si}$ & 491 & $24.9 \%$ & 845 & $42.8 \%$ \\
\hline
\end{tabular}

Fuente: Encuestas aplicadas a los estudiantes

Realizado por: Autores, 2017

\section{Análisis}

En relación a los resultados representados en el Cuadro 16, se evidencia que existe mayor presencia de parasitosis en niños que conviven con animales en casa, siendo este hábito muy importante como indicativo de transmisión de parásitos entre animales y sus dueños. Como se puede apreciar, del total de la población que dió positivo a parasitosis, el tener animales en casa aumenta el porcentaje de casos en más de un 200\%. Esta cuestión debería ser estudiada en mayor profundidad para determinar la implicación de los animales domésticos en el mantenimiento y transmisión de estos patógenos parásitos.

Cuadro 17. Cuadro de relación a si tienen animales de corral y si presenta parásitos.

\begin{tabular}{ccccc}
\hline CORRAL CON & \multicolumn{2}{c}{ CASOS } \\
ANIMALES & \multicolumn{2}{c}{ Positivo } & \multicolumn{2}{c}{ Negativo } \\
\hline No & 559 & $28.3 \%$ & 1055 & $53.5 \%$ \\
$\mathrm{Si}$ & 150 & $7.6 \%$ & 208 & $10.5 \%$ \\
\hline
\end{tabular}

Fuente: Encuestas aplicadas a los estudiantes

Realizado por: Autores, 2017 


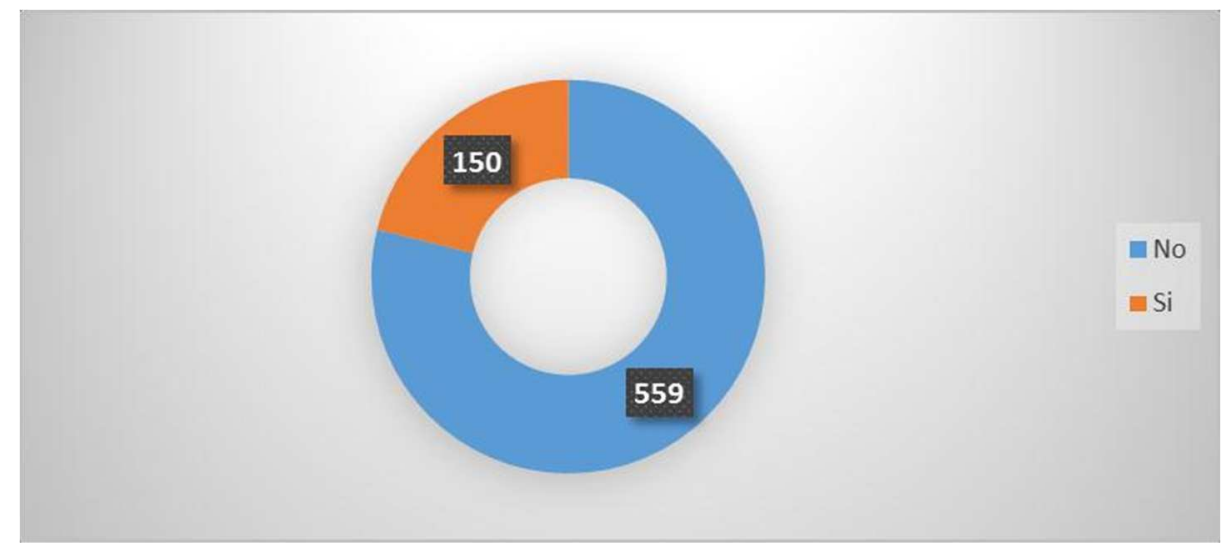

Gráfica 8. Cuadro de relación a si tienen animales de corral.

\section{Análisis}

En relación a los resultados representados en el Cuadro 17 y Gráfica 8 , se evidencia que existe menor presencia de parasitosis en niños que tienen animales de corral (7.6\% del total de casos estudiados), lo que lleva a señalar que, al situarse el corral a una determinada distancia de la casa, el grado de interacción que se requiere para que los niños adquieran infecciones parasitarias no se da con tanta intensidad como en el anterior apartado, Cuadro 16

\section{Conclusiones}

Se han identificado y clasificado los parásitos más frecuentes en la población estudiada, siendo la prevalencia total del 35.1\%. La mayor prevalencia se ha obtenido con Entamoeba coli $(26.0 \%)$, seguida de Entamoeba histolytica $(9.3 \%)$ y Giardia lamblia $(4.3 \%)$; mientras que la menor prevalencia correspondió a Trichuris trichiura $(0.1 \%)$.

Por grupos de edad se evidenció que los rangos de edades con mayor riesgo de presentar estos parásitos se sitúan entre los 5 años y los 13 años (89.3\% del total de niños parasitados), lo que podría guardar relación con factores de tipo inmunológico y conductuales por parte de los niños. El genero no se ha mostrado como un elemento determinante en la prevalencia de infecciones parasitarias.

El análisis por Unidades Educativas ha permitido determinar la existencia de diferencias en las prevalencias de parasitosis, especialmente entre Combatientes de Tapi con un $(33 \%)$ y la Unidad Educativa Camilo Gallegos Toledo (13\%). De igual modo se ha observado que las especies parásitas muestran distinta distribución en dichas unidades, lo que podría ser indicativo de la existencia de microfocos parasitarios en las distintas instituciones. 
Factores como son el ambiente de la vivienda, lugar donde defecan, limpieza o no de manos antes de comer y después de defecar, o el cuidado de las uñas han mostrado resultados inesperados en términos de porcentajes; observándose mayores porcentajes en los niños que cumplen con hábitos que deberían protegerles frente a las infecciones (ambiente urbano, defecación en baño, limpieza de manos antes de las comidas y después de defecar, y uñas cortas). Estos resultados podrían deberse a sesgos de clasificación muestral debidos a que la población se vea afectada por motivos relacionados con imagen personal.

En términos de número de casos, se ha observado que el juego en zonas de tierra se traduce en un ligero aumento de las posibilidades de contagio; especialmente con los Quistes de Giardia lamblia, Huevos de Hymenolepis nana, Quistes de Iodamoeba bütschlii y Huevos de Ascaris lumbricoides.

En los relativo a la sintomatología se observa que un importante porcentaje de los niños parasitados fueron asintomáticos $(62.2 \%$ de la población infectada); lo que avala la necesidad del diagnóstico parasitológico de estos niños, y los riesgos de recurrir exclusivamente al diagnóstico basado en la sintomatología. Los síntomas más frecuentes (fiebre, diarrea y vómitos) se han asociado a las especies parásitas Entamoeba histolytica, Giardia lamblia, Hymenolepis nana y Ascaris lumbricoides.

El agua se ha mostrado como un importante elemento de transmisión, tanto a través de su ingestión directa; donde la mayor transmisión se debe al agua de llave (37,5\% de los niños infectados), como a través del lavado de los alimentos; donde el hecho de lavar la fruta/verdura con agua de llave incrementa significativamente la probabilidad de contagio $(73.0 \%$ de $\operatorname{los}$ niños infectados).

La presencia de animales en las viviendas cohabitando con sus dueños ha incrementado notablemente el porcentaje de infecciones entre los niños $(24.9 \%$ de niños infectados con animales frente al $11.1 \%$ entre aquellos que no disponen de animales en casa). Por contrario se ha visto que la presencia de animales de corral no influye de igual forma en la infección por parásitos $(7.6 \%$ de niños infectados con animales de corral frente al $28.3 \%$ de niños infectados sin animales de corral), probablemente por la mayor distancia y separación

\section{Recomendaciones}

Se debe realizar una limpieza minuciosa en los baños ya que constituyen un importante foco de transmisión de parásitos.

Al momento de utilizar agua para el consumo humano, se recomienda hervirla hasta los $91^{\circ}$ centígrados, para matar cualquier organismo patógeno presente en ella; y evitar el empleo directo de agua de llave. 
Se recomienda que después que los niños jueguen en zonas de tierra, tener las debidas medidas de higiene necesarias para eliminar los patógenos presentes.

Antes de ingerir frutas/verduras se recomienda tratarlas con una solución de legía para reducir los organismos patógenos presentes y así evitar una contaminación.

Es importantes que se tomen los cuidados necesarios con las mascotas que tienen en casa (desparasitándolas, vacunándolas, y tenerlas limpios), para así reducir la posible transmisión de parásitos y otros organismos patógenos. De igual modo se recomienda evitar tener animales de granja (distintos de perros o gatos) en el interior de las viviendas.

Cabe añadir que estos parásitos se encontraron en una sola recolección de muestra para poder confirmar los resultados obtenidos y sean más confiables se debería realizar un examen seriado es decir por triplicado.

\section{References:}

1. Botero , D., Restrepo , M. \& Col. Parasitosis humanas Incluye animales venenosos y ponzoñosos. Quinta Edición ed. Colombia: Panamericana formas e impresos S.A., 2012. pp. 3-6.

2. Carpio, I., et al., Presence of Strongyloides stercoralis in a enteroparasites study in school students of "La Candelaria", district of Chancay, province of Huaral, department of Lima. Acta Med Per. [En linea]. 2007. (Perú). 24(3), pp. 177-180. [Consulta: 25 Dic 2015]. Disponible en: http://www.scielo.org.pe/pdf/amp/v24n3/v24n3a09.

3. Casquina, L. \& Martínez, E. Intestinal parasitism in pucchun's primary school students, prevalence and epidemiology factors, arequipa-perú 2006. Asociación Peruana de Helmintología e Invertebrados Afines (APHIA). [En linea], 2011. (Perú). 5(2), pp. 247-255. [Consulta: 25 Dic 2015]. ISSN 1995-1043. Disponible en: http://sisbib.unmsm.edu.pe/bvrevistas/neohel/v5n2/pdf/a11v5n2.pdf. pdf

4. Centro de control de enfermedades EEUU. Giardiasis. CDC [En línea]. (Estados Unidos de America). 2013. [Consulta: 8 Ene 2016]. Disponible en: http://phil.cdc.gov/PHIL_Images/8698/8698_lores.jpg

5. Cepon-Robins, T., et al., Soil-transmitted helminth prevalence and infection intensity among geographically and economically distinct Shuar communities in the Ecuadorian Amazon. The Journal of Parasitology. [En linea]. 2014. (Ecuador). 100(5), pp. 598-607. [Consulta 2015 Ago 17]. ISSN 10.1645/13-383.1. Disponible en: http://scholar.harvard.edu/files/samuel_s_urlacher/files/journal_of_pa rasitology_2014_cepon-robins.pdf 
6. Costamagna , R. \& Visciarelli, E. Parasitosis Regionales. 2 ed. Argentina, Buenos Aires: De la Universidad Nacional del Sur. 2004. pp. 13-16, 81-82, 107-109, 191-194, 229-236, 249-250, 256.

7. Gutiérrez , C., Rojas , P. \& Revollo , C. Prevalecía de Parasitosis intestinales en niños en edad escolar en los distritos 4, 5, y 6 del municipio de Tiquipaya - tercera sección provincia Quillacollo.. ESPE. [En linea], 2007.(Ecuador)., 1, pp. 1-5., [Consulta 2015 Ago 28].

Disponible

en: https://sites.google.com/a/espec.info/espec/investigacion/prevalenciade-parasitosis-intestinal- escuela-carlos-montufar.

8. Halpenny, C., et al., Regional, household and individual factors that influence soil transmitted helminth reinfection dynamics in preschool children from rural indigenous Panama. PLoS Negl Trop. [En linea], 2013. (Panama).7, p. e2070. [Consulta 2015 Ago 15]. ISSN 10.1371. Disponible

en: http://www.ncbi.nlm.nih.gov/pmc/articles/PMC3578751/.

9. Jacobsen , K. \& Ribeiro , P.Prevalencia de parasitismo intestinal en niños quechuas de zonas rurales montañosas de Ecuador. Rev Panam Salud Pública. [En linea]. 2007. (Ecuador). 23(2), pp. 125-125. [Consulta 2015 Ago 30], Disponible en: http://dx.doi.org/10.1590/S1020-49892008000200009.

10. Lujan, H.,Giardia y Giardiasis. Instituto de Investigaciones Médicas Mercedes y Martín

11. Sackey , E., Weigel , M. \& Armijos, R. Predictors and nutritional consequences of intestinal parasitic infections in rural Ecuadorian children. Journal of Tropical Pediatrics. [En linea], 2003. (Ecuador). 49, pp. 17-23. [Consulta 2015 Ago 16]. ISSN 10.1093/tropej/49.1.17. Disponible en: http://www.ncbi.nlm.nih.gov/pubmed/12630715.

12. Sanchez R y Rojas O., Intestinal parasitism and associated factors in children of the community of Santa Barbara, Venezuela. Rev Panam Infectol. [En linea], 2011. (Venezuela). 13(2), pp. 38-45. [Consulta 2015 Dec 16].Disponible en:www.revistaapi.com/wpcontent/uploads/2014/03/API_02_11_F.pdf

13. Solano , L., et al.,Influencia de las parasitosis intestinales y otros antecedentes infecciosos sobre el estado nutricional antropométrico de niños en situación de pobreza. Parasitol. latinoam. [En linea]. 2008. (Chile). 63((1-2-3-4)), pp. 12-19. [Consulta 2015 Ago 15], ISSN 0717-7712. Disponible en: http://www.scielo.cl/scielo.php?script=sci_arttext\&pid=S0717$77122008000100003 \& \operatorname{lng}=$ es.

14. Tomohissa, E., et al., Diagnosis of Ascaris lumbricoides infection using capsule endoscopy. World journal of Gastrointestinal 
Endoscopy. [En linea],2013. (Brazil). 5(4), pp. 189-190. [Consulta 2015 Ago 15]. ISSN 10.4253/wjge.v5.i4.189. Disponible en: http://www.wjgnet.com/1948-5190/pdf/v5/i4/189.pdf

15. Vasquez, O. \& Campos, T.Giardiasis. La parasitosis mas frecuente a nivel mundial. Rev. DelCentro de Inv. [En linea]. 2009. (Mexico). 8(31), pp. 75-90., [Consulta: 6 Ene 2016]. ISSN 1938-3207. Disponible en: http://www.redalyc.org/pdf/342/34211305006.pdf 